\title{
Erratum: Quantum State Discrimination Using the Minimum Average Number of Copies [Phys. Rev. Lett. 118, 030502 (2017)]
}

Sergei Slussarenko, Morgan M. Weston, Jun-Gang Li, Nicholas Campbell, Howard M. Wiseman, and Geoff J. Pryde

(Received 30 May 2017; published 23 June 2017)

DOI: 10.1103/PhysRevLett.118.259901

There was a typographical error in Eq. (4) of the Letter. The +1 term should be omitted and the equation should read

$$
C^{\mathrm{LOL}}(\varepsilon)=\left\lceil\frac{\ln \left(\varepsilon-\varepsilon^{2}\right)-\ln \left(q_{1} q_{2}\right)}{2 \ln (\cos 2 \theta)}\right\rceil,
$$

as in Eq. (S18) in the Supplemental Material. This error is purely typographical and not present in any of the calculations, nor in any of the other equations in the Letter and the Supplemental Material. The conclusions and results of the Letter remain unchanged.

We thank Dongxu Chen from Xi' an Jiaotong University for bringing this to our attention. 